\title{
PREPARATION AND CHARACTERIZATION OF SOLID DISPERSIONS OF CARVEDILOL WITH POLOXAMER 188
}

\author{
ANSHU SHARMA ${ }^{* *}$, CHANDRA PRAKASH JAIN ${ }^{2}$, YUVERAJ SINGH TANWAR ${ }^{1}$ \\ 'Bhupal Nobles' College of Pharmacy, Udaipur, Rajasthan, India. \\ ${ }^{2}$ Department of Pharmaceutical Sciences, MohanLal Sukhadia University, Udaipur, India.
}

(Received: August 2, 2011 - Accepted: November 1, 2012)

\begin{abstract}
Solid dispersions in water-soluble carriers have attracted considerable interest as a means of improving the dissolution rate, and hence possibly bioavailability, of a range of hydrophobic drugs. The poor solubility of carvedilol leads to poor dissolution and hence variation in bioavailability. The purpose of the present investigation was to increase the solubility and dissolution rate of carvedilol for enhancement of oral bioavailability. In the present investigation solid dispersions with poloxamer 188 were prepared by fusion method. The physical mixture and solid dispersion(s) were characterized for drug-carrier interaction, drug content, solubility and dissolution rate. The solubility of drug increased with increasing polymer concentration. The dissolution rate was substantially improved for carvedilol from its solid dispersion compared with pure drug and physical mixture. As indicated from X-ray diffraction pattern, DSC thermograms and SEM photographs, carvedilol was in the amorphous form, which confirmed the better dissolution rate of solid dispersions. FTIR results proved no chemical interaction between carvedilol and poloxamer 188. SEM images showed a novel morphology of solid dispersion compared with pure drug. The solid dispersion was stable under accelerated storage conditions.
\end{abstract}

\section{INTRODUCTION}

Solid dispersion represents a useful pharmaceutical technique for increasing the dissolution, absorption and therapeutic efficacy of drugs in dosage forms ${ }^{1}$, ${ }^{2}$. The formulation of poorly soluble compounds for oral delivery now presents one of the greatest and most frequent challenges to formulation scientists in the pharmaceutical industry ${ }^{3}$.

The use of solid dispersions of drugs in water soluble carriers, in which the drugs are highly soluble; to increase the dissolution rate and bioavailability of poorly soluble drugs have been studied extensively. This technique provides a means of reducing particle size to nearly a molecular level. As the soluble carrier dissolves, the insoluble drug is exposed to the dissolution medium as very fine particles for quick dissolution and absorption ${ }^{4,5}$.

Literature shows that the fusion method is technically less difficult method of preparing solid dispersions and has been frequently used for the preparation of solid dispersions for dissolution enhancement ${ }^{6-9}$. Earlier studies show that solid dispersion systems increased the drug dissolution due to improved solubility wettability and dispersibility using hydrophyllic carriers ${ }^{10-12}$.

Carvedilol is a multiple-action cardiovascular drug that is currently approved in many countries for the treatment of hypertension (Fig. 1). The reduction in blood pressure produced by carvedilol results primarily from beta-adrenoceptor blockade and vasodilation, the latter resulting from alpha 1 -adrenoceptor blockade ${ }^{13-15}$. These actions as well as several other carvedilol activities are associated with cardioprotection in animal models that occurs to a degree that is greater than that observed with other drugs. The multiple actions of carvedilol may also provide the underlying rationale for the use of the drug in the treatment of coronary artery disease and congestive heart failure. Carvedilol is well absorbed from the gastrointestinal tract but is subject to considerable first-pass metabolism in the liver; its absolute bioavailability is about $25 \%$. Being categorized as class II compound as per the BCS classification system, it posses very poor bioavailability and shows significant first- pass metabolism ${ }^{16-18}$.

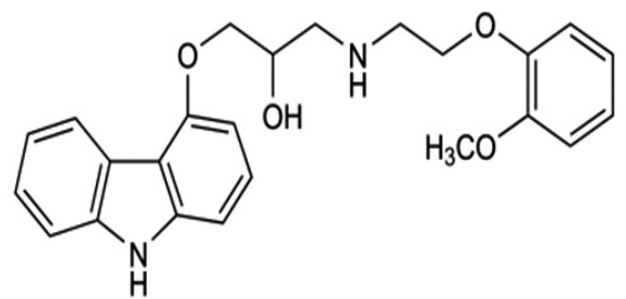

Figure 1. Chemical structure of carvedilol.
Poloxamer are water soluble non-ionic surface active block copolymers consisting of both lipophilic polyoxypropylene and hydrophilic polyoxyethylene chains. The differences in the length of the polyoxyethylene and the polyoxypropylene chains in the different products are responsible for the divergencies in their physical and chemical properties. Poloxamer 188 containing $80 \%$ ethylene oxide has been used in solid dispersion to improve drug solubility ${ }^{19,20}$. The present study is aimed to prepare and characterize the solid dispersion of carvedilol with poloxamer 188 using fusion method in order to improve its aqueous solubility and dissolution rate for enhancement of oral bioavailability.

\section{EXPERIMENTAL}

\section{Materials}

Carvedilol was provided by Sun Pharmaceutical Ltd., Baroda, India and Poloxamer 188 from BASF Corporation, Mumbai, India as a gift sample. All other chemicals and reagents used were of analytical grade.

Preparation of Physical Mixtures and Solid Dispersions of Carvedilol

The drug and carriers in ratio of 1:1, 1:3, 1:5 (PM1, PM2, PM3) were mixed thoroughly in a mortar with pestle and passed through a 60-mesh (250 $\mu \mathrm{m})$ screen to obtain physical mixtures (PMs). Solid dispersions (SDs) of carvedilol in poloxamer 188 containing different weight ratio 1:1, 1:3, 1:5 (SD1, SD2, SD3) were prepared by fusion method. Poloxamer 188 was heated at $60{ }^{\circ} \mathrm{C}$ in an oil bath, until it melted completely. The drug was added to the molten polymer and mixed thoroughly in mortar with pestle. The dispersion was cooled to ambient conditions, milled, and passed through a 40-mesh (425 $\mu \mathrm{m})$ screen.

Characterization of Physical Mixtures and Solid Dispersions of Carvedilol

\section{Phase Solubility Studies}

Solubility measurements were performed according to method reported by Higuchi and Connors ${ }^{21}$. An excess amount of the drug was added to $10 \mathrm{ml}$ volumetric flask containing $10 \%, 20 \%, 30 \%, 40 \%$ aqueous solution of carriers. The samples were allowed to shake for 48 hours at $25 \pm 1{ }^{\circ} \mathrm{C}$. The solutions were filtered through membrane filter $(0.45 \mu)$. After 48 hours, the carvedilol concentration was determined spectrophotometrically at $285 \mathrm{~nm}$. Solubility studies of physical mixtures and solid dispersion was also performed in same manner.

Flow Properties

The flow properties of PMs and SDs were characterized in terms of angle of repose, Carr index and Hausner ratio ${ }^{22}$. For determination of angle of repose 
$(\theta)$, the sample was poured through the walls of a funnel, which was fixed at a position such that its lower tip was at a height of exactly $2.0 \mathrm{~cm}$ above hard surface. The sample was poured till the time when upper tip of the pile surface touched the lower tip of the funnel. The $\tan ^{-1}$ of the (height of the pile / radius of its base) gave the angle of repose ${ }^{23}$.

Sample was poured gently through a glass funnel into a graduated cylinder cut exactly to $10 \mathrm{ml}$ mark. Excess sample was removed using a spatula and the weight of the cylinder with pellets required for filling the cylinder volume was calculated. The cylinder was then tapped from a height of $2.0 \mathrm{~cm}$ until the time when there was no more decrease in the volume. Bulk density $(\rho b)$ and tapped density ( $\rho t)$ were calculated. Hausner ratio (HR) and Carr index (IC) were calculated according to the two equations given below:

$$
\begin{aligned}
& \mathrm{HR}=\rho \mathrm{t} / \rho \mathrm{b} \\
& \mathrm{IC}=(\rho \mathrm{t}-\rho \mathrm{b}) / \rho \mathrm{t}
\end{aligned}
$$

\section{Particle Size Analysis}

The size distribution in terms of average diameter $\left(\mathrm{d}_{\text {avg }}\right)$ of the powder of physical mixtures and solid dispersions was determined by an optical microscopic method. A compound microscope (Olympus NWF 10X Educational Scientific Stores, India) fitted with a calibrated ocular micrometer and a stage micrometer slide was used to count at least 100 particles.

\section{Drug Content}

SDs and PMs of carvedilol were tested for drug content uniformity. Accurately weighed amount of sample was dissolved in $10 \mathrm{ml}$ of methanol and stirred on magnetic stirrer for 10 minutes. The solution was filtered through membrane filter $(0.45 \mu)$, diluted suitably and assayed for carvedilol content spectrophotometrically at $285 \mathrm{~nm}^{24}$.

\section{Scanning Electron Microscopy (SEM)}

The SEM analysis was carried out using scanning electron microscope (S3400, Hitachi, JAPAN). Prior to examination, samples were mounted on an aluminum stub using a double sided adhesive tape and then making it electrically conductive by coating with a thin layer of gold (approximately $24 \mathrm{~nm}$ ) in vacuum. The scanning electron microscope was operated at an acceleration voltage of $15 \mathrm{KV}$.

\section{Differential Scanning Calorimetry (DSC)}

Differential Scanning Calorimetry (DSC) analysis of the samples was carried out on a Perkin-Elmer DSC7. Samples $(6.5-10 \mathrm{mg})$ were heated under nitrogen atmosphere on an aluminum pan at a heating rate of $10{ }^{\circ} \mathrm{C} / \mathrm{min}$ over the temperature range of 5 and $300{ }^{\circ} \mathrm{C}$. DSC analysis was carried out under nitrogen gas flow of $20 \mathrm{lb} / \mathrm{in}^{2}$.

\section{Powder X- Ray Diffraction (PXRD)}

PXRD patterns were recorded using Philips PW 1729 X- ray generator, USA fitted with a copper target, a voltage of $40 \mathrm{kV}$, and a current of $30 \mathrm{~mA}$. The scanning rate was $1^{\circ} / \mathrm{min}$ over a $2 \theta$ range of $1-50^{\circ}$. Powder X- ray diffraction patterns were traced for carvedilol, physical mixture and solid dispersions. The samples were slightly ground and packed into the aluminum sample container.

\section{Fourier Transform Infrared Spectroscopy (FTIR)}

Fourier transform infrared spectra were obtained using Shimadzu FTIR8400 S spectrometer, Japan. Samples of carvedilol, poloxamer 188, physical mixtures and solid dispersions were ground and mixed thoroughly with potassium bromide at a 1:5 sample $/ \mathrm{KBr}$ ratio. The $\mathrm{KBr}$ discs were prepared by compressing the powders at a pressure of $5 \mathrm{~T}$ for $5 \mathrm{~min}$ in a hydraulic press. The scanning range was 40 to $4000 \mathrm{~cm}^{-1}$ and the resolution was $4 \mathrm{~cm}^{-1}$.

\section{Dissolution Studies}

The release rate of carvedilol from solid dispersions was determined using United States Pharmacopeia (USP) Dissolution Testing Apparatus 2 (paddle method; Veego Scientific, Mumbai, India). The dissolution test was performed using $900 \mathrm{ml}$ of distilled water, at $37 \pm 0.5^{\circ} \mathrm{C}$ and $50 \mathrm{rpm}$ for 2 hours ${ }^{25}$. Samples equivalent to $12.5 \mathrm{mg}$ of carvedilol were taken for dissolution studies. A sample $(10 \mathrm{ml})$ of the solution was withdrawn from the dissolution apparatus 10 minute interval and the samples were replaced with fresh dissolution medium. The samples were filtered through a $0.45 \mu$ membrane filter and diluted to a suitable concentration with distilled water. Absorbance of these solutions was measured at $285 \mathrm{~nm}$ using a UV/Vis double-beam spectrophotometer (Shimadzu 1700, Japan).

\section{Stability Studies}

The accelerated stability study of prepared solid dispersion was carried out at $40^{\circ} \mathrm{C} / 75 \% \mathrm{RH}$ for a period of up to 3 month $\mathrm{s}^{26}$. An accurately weighed amount of sample was placed into glass vials with aluminum-lined caps and stored in microprocessor controlled humidity chamber; samples were characterized as a function of exposed time. The samples were removed and evaluated for flow properties, particle size, solubility, drug content, dissolution, PXRD, DSC and FTIR studies. The differences in parameters from solid dispersion were evaluated using unpaired t-test. In t-test, a probability value of $\mathrm{p}<0.05$ was considered to be statistically significant.

\section{RESULTS AND DISCUSSION}

Physical mixtures and solid dispersions were evaluated for particle size, solubility, drug content, flow properties, surface morphology, crystallinity and dissolution studies. The result of physicochemical characterization of physical mixtures and solid dispersions of carvedilol are summarized in Table 1.

\section{Phase Solubility Studies}

The solubility of carvedilol in distilled water at $25{ }^{\circ} \mathrm{C}$ was found to be $0.002 \mathrm{mg} / \mathrm{ml}$. At $40 \% \mathrm{w} / \mathrm{v}$ of poloxamer 188 the increase in solubility at $25^{\circ} \mathrm{C}$ was 22 fold compared with pure drug.

The effect of poloxamer 188 upon the solubility of carvedilol is presented in Fig. 2. The increase in solubility was linear with respect to the weight fraction of the carrier. The increase in solubility with increasing poloxamer concentration indicates the solvent properties of poloxamer 188 for the drug. Poloxamer 188 causes a decrease of interfacial tension between the drug and dissolution medium. This behaviour suggests the feature of $A_{L}$ type solubility phase diagram. This finding is in accordance with Zidan et al. regarding the increased solubility of rofecoxib ${ }^{27}$. The solubility of carvedilol in solid dispersion SD1, SD2, SD3 was found to enhance by $36,54,78$ folds respectively in comparison with pure drug. These results could explain that the reduction in crystallinity of drug led to a decrease of the energy required in the dissolving process and also to a highly dispersed state of the drug.

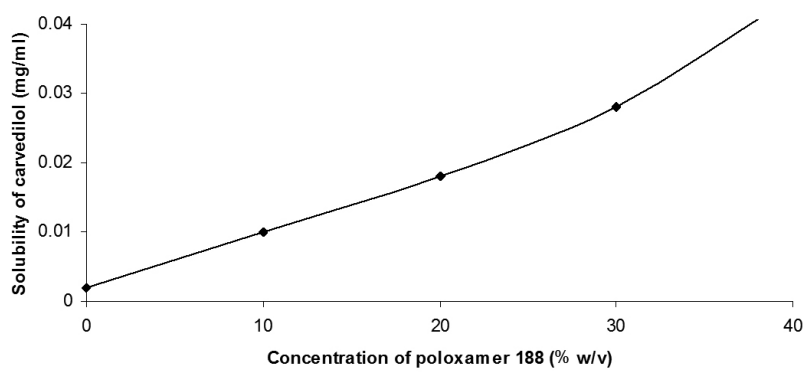

Figure 2. Solubility curve of carvedilol with poloxamer 188.

\section{Flow Properties}

SDs and PMs were evaluated for their flow properties. Angle of repose was in the range of 50.512 to 58.528 . Carr index was found to be 0.362 to 0.421 and Hausner ratio ranged from 1.580 to 1.743 . The values of angle of repose, carr index and hausner ratio indicate that the particles of physical mixtures and solid dispersions exhibited poor flow properties which suggest addition of glidants during formulation of solid dosage form employing solid dispersion of carvedilol.

\section{Particle Size Analysis}

Particle size was determined by optical microscopy. It was found to be 61.21 to $67.38 \mu \mathrm{m}$ for PMs and 33.47 to $37.14 \mu \mathrm{m}$ for SDs. The particles of solid dispersion were smaller than PMs when observed by optical microscopy. This indicates that reduction in particle size may also be factor influencing solubility of drug in physical mixture and solid dispersion.

\section{Drug Content}

The drug content was found in the range of 98.161 to $99.814 \%$ indicating the acceptability of fusion method for preparation of solid dispersions. Drug content was found to be uniform among all physical mixtures and solid dispersion. Low values of standard deviation in drug content of PMs and SDs indicated uniform drug distribution in all the prepared batches. Therefore, the method used in this study appears to be reproducible for the preparation of solid dispersions. 
Table 1. Physicochemical characterization of PMs and SDs of carvedilol.

\begin{tabular}{|c|c|c|c|c|c|c|c|}
\hline Code & Ratio & $\begin{array}{c}\text { Average } \\
\text { Particle Size } \\
(\mu \mathrm{m})\end{array}$ & $\begin{array}{l}\text { Solubility } \\
(\mathrm{mg} / \mathrm{ml})\end{array}$ & Drug content $(\%)$ & Angle of repose $(\theta)$ & $\begin{array}{c}\text { Hausner ratio } \\
\qquad\left(H_{R}\right)\end{array}$ & $\begin{array}{c}\text { Carr index } \\
\text { (IC) }\end{array}$ \\
\hline PM1 & $1: 1$ & $61.21 \pm 0.96$ & $0.015 \pm 0.003$ & $98.814 \pm 0.376$ & $50.512^{\circ} \pm 0.290^{\circ}$ & $1.631 \pm 0.001$ & $0.391 \pm 0.001$ \\
\hline PM2 & $1: 3$ & $64.20 \pm 1.21$ & $0.028 \pm 0.002$ & $99.680 \pm 0.679$ & $54.210^{\circ} \pm 0.352^{\circ}$ & $1.743 \pm 0.000$ & $0.421 \pm 0.004$ \\
\hline PM3 & $1: 5$ & $67.38 \pm 2.31$ & $0.04 \pm 0.007$ & $99.729 \pm 1.129$ & $58.528^{\circ} \pm 0.235^{\circ}$ & $1.675 \pm 0.002$ & $0.405 \pm 0.003$ \\
\hline SD1 & $1: 1$ & $33.47 \pm 0 . .93$ & $0.072 \pm 0.004$ & $98.161 \pm 0.850$ & $56.389^{\circ} \pm 0.670^{\circ}$ & $1.580 \pm 0.001$ & $0.362 \pm 0.001$ \\
\hline $\mathrm{SD} 2$ & $1: 3$ & $35.36 \pm 0.23$ & $0.108 \pm 0.003$ & $98.614 \pm 1.294$ & $55.462^{\circ} \pm 0.850^{\circ}$ & $1.610 \pm 0.001$ & $0.380 \pm 0.002$ \\
\hline SD3 & $1: 5$ & $37.14 \pm 1.09$ & $0.156 \pm 0.002$ & $99.262 \pm 1.035$ & $54.653^{\circ} \pm 0.784^{\circ}$ & $1.591 \pm 0.001$ & $0.371 \pm 0.002$ \\
\hline
\end{tabular}

Scanning Electron Microscopy (SEM)

SEM photomicrographs of pure drug, poloxamer 188, physical mixture and solid dispersion are depicted in Fig. 3. SEM of carvedilol and poloxamer 188 showed crystal form and globular form respectively. In solid dispersion the structure of carvedilol crystal or mixture is completely different. This indicates that a kind of new structure formed in SD of Poloxamer 188. These findings demonstrated that the drug was thoroughly mixed in the carriers with the loss of little crystallinity. The similar microscopic photographs of SDs might be attributed to the similar intermolecular interaction between drug and carrier which was confirmed in the PXRD and DSC studies.

In case of pure carvedilol rod shaped crystals can be seen, whereas in SEM of poloxamer 188, large globular particles are seen. In case of SDs, it was difficult to distinguish the presence of carvedilol crystals. Carvedilol crystals appeared to be incorporated into the particles of the polymers. The solid dispersion looked like a matrix particle. The results could be attributed to dispersion of the drug in the molten mass of the polymer.
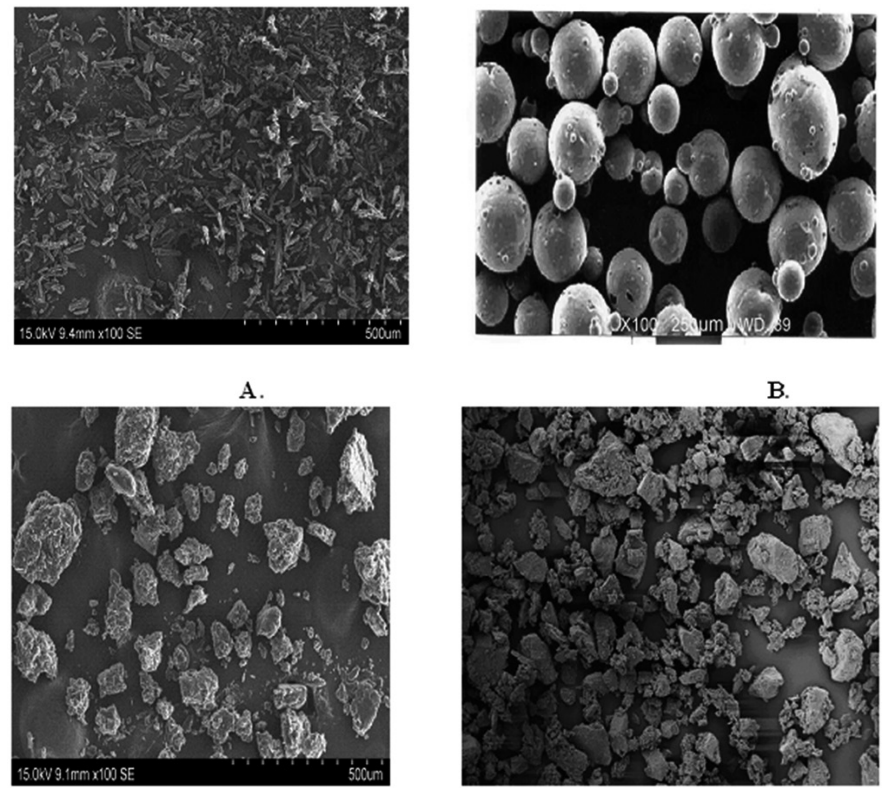

C.

D.

Figure 3. SEM of (A) Carvedilol, (B) Poloxamer 188, (C) Physical mixture and (D) Solid dispersion.

Differential Scanning Calorimetric Studies (DSC)

Differential scanning calorimetry enables the quantitative detection of all processes in which energy is required or produced (i.e., endothermic and exothermic phase transformations). The thermal behavior was studied using differential scanning calorimetry in order to confirm the formation of solid dispersions. The thermograms for pure carvedilol and poloxamer 188, and SD and physical mixture are presented in Fig. 4. The carvedilol showed a melting endotherm at $115^{\circ} \mathrm{C}$ whereas pure poloxamer 188 showed a melting endotherm at $30{ }^{\circ} \mathrm{C}$. The physical mixture formulation of carvedilol and poloxamer 188 also showed no endothermic peak of carvedilol, even though the peaks derived from carvedilol were observed in XRD.

Thermograms of solid dispersion showed the absence of a carvedilol peak, suggesting that carvedilol is completely soluble in the liquid phase with poloxamer 188. However, the melting peak of poloxamer in solid dispersion was observed at slightly lower temperatures (between $27^{\circ} \mathrm{C}-30^{\circ} \mathrm{C}$ ) than that of pure poloxamer $188\left(30^{\circ} \mathrm{C}\right)$, indicating the miscibility of the drug in carrier. It is speculated that carvedilol is dissolved in melted poloxamer 188 during the DSC measurement, and only one endothermic peak at around $30^{\circ} \mathrm{C}$, which corresponds to the melting of poloxamer 188 , was observed. This finding is in agreement with the report of Yamashita et al., which reported the absence of the endothermic peak of tacrolimus in the physical mixture of tacrolimus with PEG $6000^{28}$

Reduction in intensity and shifting of sharp melting peak of drug in solid dispersion indicates that the degree of crystallinity is considerably reduced and the drug is present in an amorphous form. The endothermic peak corresponding to melting of carvedilol was absent in the DSC thermogram of solid dispersion. It might be due to the presence of the amorphous form of carvedilol in the solid dispersion or the dissolution of crystalline carvedilol into the molten carrier.

\section{Powder X- Ray Diffraction Studies}

Powder X-ray diffraction analysis can be used to judge any changes in crystallinity of the drug which precipitated in an amorphous form, when formulated into a solid dispersion. PXRD could be used to study any changes in crystallinity of the drug which could be one of the mechanisms responsible for improved dissolution.

XRD analysis was performed to confirm the results of DSC studies. The X-ray diffractograms of pure carvedilol and poloxamer 188, physical mixture and solid dispersion are shown in Fig. 5.

Numerous diffraction peaks of carvedilol were observed at $2 \theta$ of $12.8^{\circ}$, $15.62^{\circ}, 17.46^{\circ}, 18.56^{\circ}, 20.1^{\circ}, 24.3^{\circ}, 26.2^{\circ}$ indicating the presence of crystalline carvedilol. Poloxamer 188 is crystalline in nature and gives two characteristic peaks: one at $19^{\circ}$ and the other broader one between $22^{\circ}$ and $23^{\circ}$. It was confirmed that the crystallinity of the carvedilol does not change in the physical mixture with carrier. The PXRD of solid dispersion exhibited the absence of characteristic peaks of carvedilol, suggesting that carvedilol is completely soluble in the liquid phase with poloxamer 188 and confirming that carvedilol, is in amorphous form with SDs. The XRD and DSC studies indicated that the transformation of crystalline carvedilol (in pure drug) to amorphous carvedilol 
(in solid dispersions) by the solid dispersion technology.

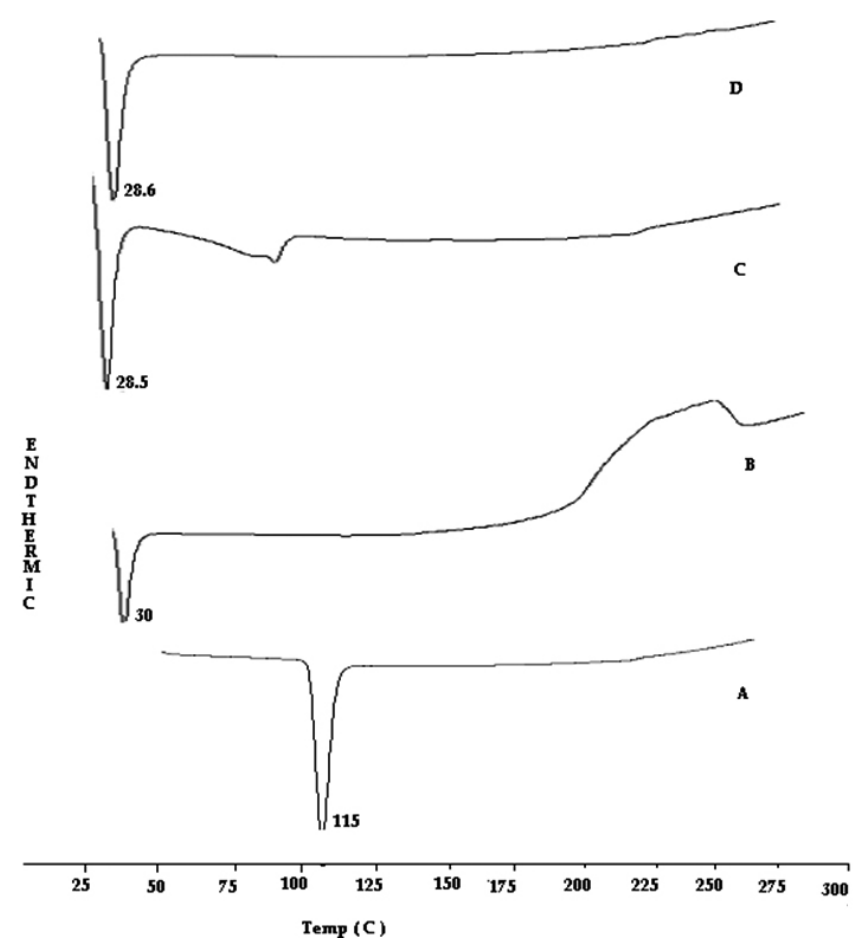

Figure 4. DSC thermogram of (A) Carvedilol, (B) Poloxamer 188, (C) Physical mixture and (D) Solid dispersion.
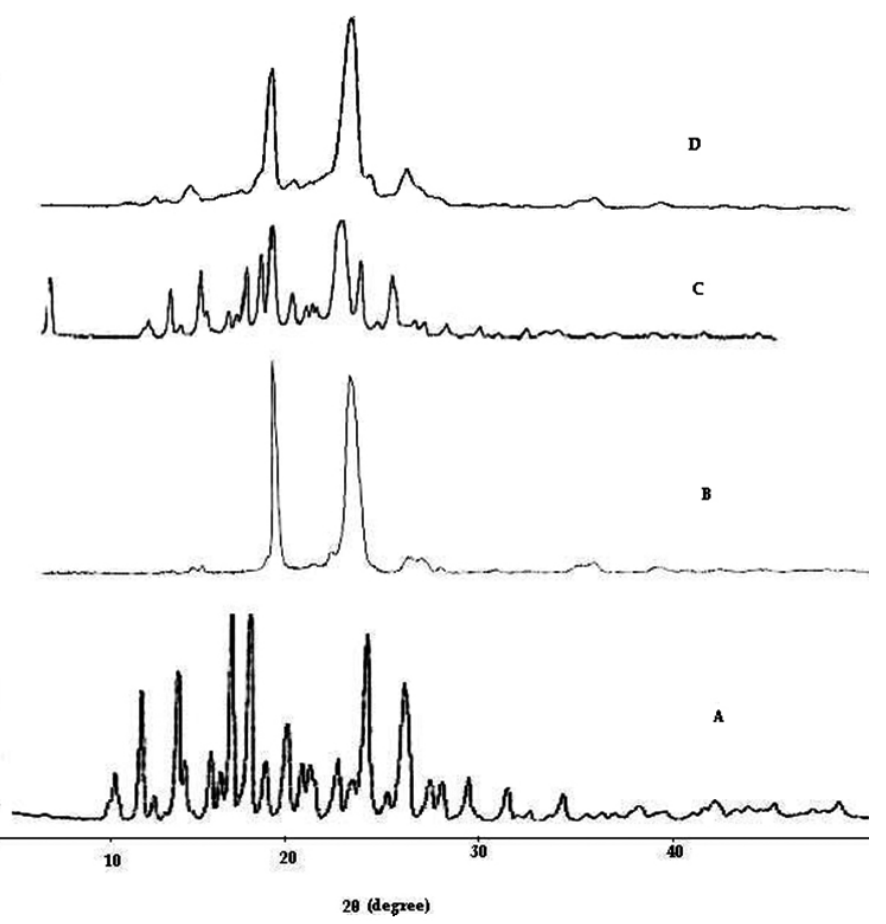

Figure 5. Powder X-ray diffraction pattern of (A) Carvedilol, (B) Poloxamer 188, (C) Physical mixture and (D) Solid dispersion.

Fourier Transform Infrared Spectroscopy (FTIR)

FTIR spectroscopy analysis was done to analyze physico-chemical interactions between carvedilol and poloxamer 188 in form of solid dispersions. Fig. 6 represents the FTIR spectra and characteristic wave numbers of carvedilol, poloxamer 188, and two compositions. The IR spectrum of carvedilol (Fig. 6a) is characterized by principal absorption peaks at characteristic peaks at 3346.27 $\mathrm{cm}^{-1}$ (O-H and N-H stretching vibration peaks merged together), 2925.81 $\mathrm{cm}^{-1}$ (C-H stretching vibrations), $1598.88 \mathrm{~cm}^{-1}$ (N-H bending vibrations) and $1253.64 \mathrm{~cm}^{-1}$ (O-H bending and $\mathrm{C}-\mathrm{O}$ stretching vibrations).

The IR spectrum of poloxamer 188 (Fig. 6b) is characterized by principal absorption peaks at $2883 \mathrm{~cm}^{-1}$ (C-H stretch aliphatic), $1341 \mathrm{~cm}^{-1}$ (in-plane O-H bend) and $1099 \mathrm{~cm}^{-1}$ (C-O strech), which were consistent in all binary systems with the drug.

The IR spectrum of the physical mixture (Fig. 6c) displayed the superimposition pattern of carvedilol and polymer peaks with decreased peak intensity and little shifting of the peaks. The IR spectrum of 1:5 solid dispersion prepared by fusion method (Fig. 5d) shows disappearance of peaks at 3341 $\mathrm{cm}^{-1}$ and the presence of all other carvedilol peaks with decreased intensity. In all other carvedilol peaks were smoothened, indicating a strong physical interaction of carvedilol with polymer. However, no additional peak was observed in any binary system, indicating absence of any chemical interaction between carvedilol and polymer.

As such, the FTIR spectra of carvedilol/poloxamer 188 compositions did not show significant shifts suggestive of an interaction. Instead, the spectra show few to no changes in the absorption bands characteristic of carvedilol. The study indicates that carvedilol has strong physical interaction with poloxamer 188 in solid state. FTIR spectroscopy revealed the possibility of inter-molecular hydrogen bonding in solid dispersions.

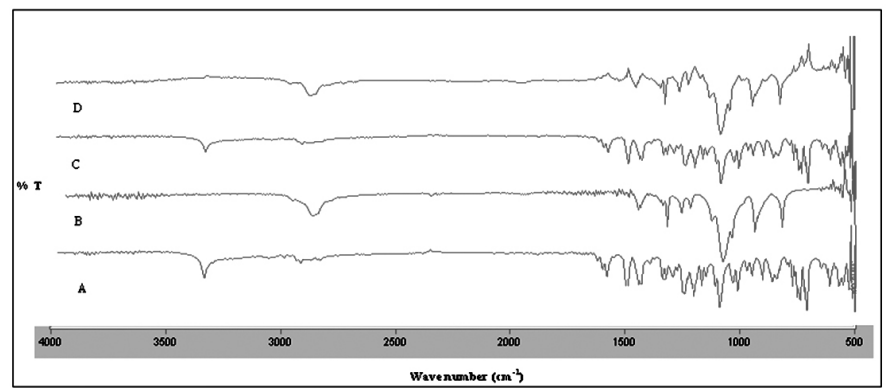

Figure 6. FTIR spectra (A) Carvedilol, (B) Poloxamer 188, (C) Physical mixture and (D) Solid dispersion.

\section{Dissolution Studies}

The in vitro dissolution profiles of the pure drug, various solid dispersions using poloxamer 188 , and their respective physical mixtures in distilled water for 120 minutes are shown in Fig. 7. At the end of 120 minutes, $42.6 \%, 47.92$ $\%, 53.81 \%, 76.60 \%, 86.186 \%$ and $93.214 \%$ carvedilol was released from pure drug sample, PM1, PM2, PM3, SD1, SD2, and SD3 respectively. All of the physical mixture and solid dispersion samples showed improved dissolution of carvedilol over pure drug. All of the solid dispersion samples revealed more improved carvedilol dissolution than their respective physical mixture samples. This observation indicated that the increased dissolution of carvedilol from solid dispersion due to presence of drug in amorphous state as compared the physical mixtures and pure drug, where drug is present in crystalline state.

Both physical mixtures and solid dispersions showed enhanced dissolution rate as compared with pure drug. Physical mixtures increased the solubility and maximizing the surface area of the drug that came in contact with the dissolution medium as the carrier dissolved. This might due to the surface tension lowering effect of polymer to the medium, resulting in wetting of hydrophobic drug of crystalline surface. Several mechanisms may be possible for the enhanced release of carvedilol in solid dispersion formulation with water soluble polymeric surfactant poloxamer 188 . This can be attributed to the reduction of crystallinity of drug resulting in improved release (supported by X-ray diffraction); reduction of particle size to expand the surface area for dissolution ${ }^{29}$. The initial high drug release is observed at the 10-min time point, and gets reduced at subsequent time points. This may be because the equilibrium concentration that can be achieved with the given formulation in the solution is less than what was achieved at the first dissolution time point. Probably the initial rapid flux of the drug from the solid dispersion particles to the dissolution medium resulted in a high concentration, which got reduced with time. Slow dissolution was observed subsequently till the equilibrium concentration was reached ${ }^{30}$. 


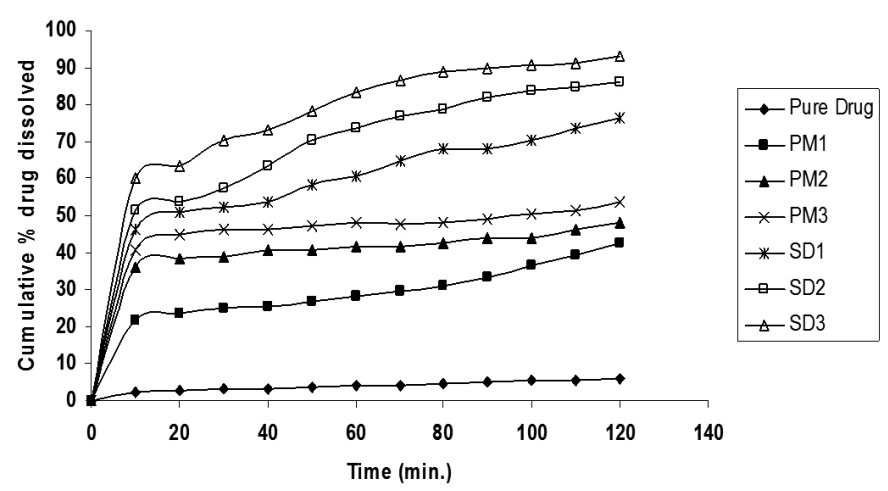

Figure 7. Dissolution profile of pure drug, physical mixtures and solid dispersions of carvedilol.

\section{Stability Studies}

The purpose of stability testing is to provide evidence on how the quality of a drug substance or drug product varies with time under the influence of a variety of environmental factors such as temperature and humidity. Stability studies were carried out for the solid dispersions obtained by fusion method by exposing them to $40^{\circ} \mathrm{C} / 75 \% \mathrm{RH}$. The polymer in an amorphous solid dispersion is used to stabilize the amorphous drug so as to prevent crystallization and to provide stability over time and under a variety of stress conditions, such as elevated temperature and relative humidity $(\mathrm{RH})^{31}$. The flow properties, particle size, drug content, solubility, dissolution, PXRD, DSC and FTIR studies were carried out at the end of 3 months and compared to day zero. The results do not show any significant change $(p>0.05)$ in the flow properties, particle size, solubility, drug content and dissolution rate of solid dispersion in comparison with initial values when stored in different storage condition. Also, the FTIR, DSC and PXRD patterns of the solid dispersion recorded after 3 months in different stress conditions gave identical patterns to the initial ones indicated that drug was stable in the solid dispersion.

\section{CONCLUSION}

The solid carrier played a significant role in the initial enhancement of drug dissolution in our studies. A remarkable increase in both the solubility and dissolution of carvedilol were observed in all carvedilol solid dispersions as compared with pure carvedilol and their physical mixtures. The rate of the dissolution of carvedilol from solid dispersion depended on the concentration of the carrier. Dissolution of drug increased with an increase in carrier content. A high proportion of poloxamer 188 in the solid dispersion significantly affected the improvement in the dissolution rate. PXRD, DSC, SEM and FTIR results confirmed the amorphous state of drug in solid dispersion. The above studies indicate that poloxamer 188 inhibited the crystallization of drugs, resulting in the amorphous state form of the drug in solid dispersion. In the stability study, no significant changes were recorded with respect to flow properties, particle size, drug content, solubility, dissolution rate, PXRD, DSC and FTIR over a period of 3 months. The solid dispersion technique with poloxamer 188 as a carrier provides a promising way to enhance the solubility and dissolution rate of carvedilol.

\section{REFERENCES}

1. M. Mayersohn, M. Gibaldi, J. Pharm. Sci., 55, 1323, (1966).

2. W. L. Chiou, S. J. Riegelman, J. Pharm. Sci., 60, 1281, (1971).

3. G. L. Amidon, H. Lennernas, V. P. Shah, J. R. Crison, Pharm. Res., 12, 413, (1995).

4. S. R. Vippagunta, Z. Wang, S. Hornung, S. L. Krill, J. Pharm. Sci., 96, 294, (2006).

5. F. Damian, N. Blaton, R. Kinget, G. Van den Mooter, Int. J. Pharm., 244, 87, (2002).

6. J. W. McGinity, P. Maincent, H. Steinfink, J. Pharm. Sci., 73, 1441, (1984).

7. D. J. Greenhalgh, A. C. Williams, P. Timmins, P. York, J. Pharm. Sci., $88,1182,(1999)$

8. M. C. Gohel, L. D. Patel, Drug Dev. Ind. Pharm., 29, 299, (2003).

9. G. F. Palmeiri, F. Cantalamessa, P. D. Martino, C. Nasuti, S. Martelli, Drug Dev. Ind. Pharm., 28, 1241, (2002).

10. J. W. Lu, M. Z. Wang, P. Ding, Z. S. Pan. Chinese Pharm. J., 30, 23,
(1995).

11. J. L. Ford, Pharm. Acta Heh., 61, 69, (1986).

12. R. R. Ruffolo, D. A. Boyle, D. P. Brooks, Cardiovasc. Rev., 10, 127, (1992).

13. Y. S. Tanwar, C.S. Chauhan, A. Sharma, Acta Pharm., 57, 151, (2007).

14. C. J. Dunn, A.P. Lea, A. J. Wagstaff, Drugs. 54, 161, (1997).

15. N. A. Kasim, M. Whitehouse, C. Ramachandran, M. Bermejo, H. Lennernals, A. S. Hussain, H. E Junginger, S. A. Stavchansky, K. K. Midha, V. P. Shah, G. L. Amidon, Mol. Pharm. 1, 85, (2004).

16. R. R. Jr. Ruffolo, G. Z. Feuerstein. Cardiovasc. Drugs Ther., 11, 247, (1997).

17. K. E. Thummel, D. D. Shen, Goodman and Gilman's The Pharmacological Basis of Therapeutics, New York, Mc. Graw Hill, 2001.

18. S. C. Shin, C. W. Cho, Pharm. Dev. Tech., 2, 403, (1997).

19. S. R. Vippagunta, K. A. Maul, S. Tallavajhala, D. J. W. Grant, Int. J. Pharm., 236, 111, (2002).

20. A. Wade, P. J. Weller, Handbook of Pharmaceutical Excipients, Washington DC, American Pharmaceutical Association, 1994.

21. T. Higuchi, K. A. Connors, Adv. Anal. Chem. Instr., 4, 117, (1965).

22. V. R. Sinha, M. K. Agarwal, R. Kumria, Curr. Drug Deliv., 2, 1, (2005).

23. M. Jaimini, A. C. Rana, Y. S. Tanwar, Curr. Drug Deliv., 4, 51, (2007).

24. H. Thierry, P. Geraldine, Eur. J. Pharm. Sci., 15, 347, (2002).

25. United States Pharmacopoeia 24, National Formulary 19, Vol. II, USP Convention, Rockville. 2000; pp. 1941-1944.

26. V. Pokharkar, A. Khanna, V. Venkatpurwar, S. Dhar, L. Mandpe, Acta Pharm., 59, 121, (2009).

27. A. S. Zidan, O. A. Sammour, M. A. Hammad, N. A. Megrab, AAPS PharmSci Tech., 7, E1, (2006).

28. K. Yamashita, T. Nakate, O. Kazuto, A. Ohike, Y. Tokunaga, R. Ibuki, K. Higaki, T. Kimura, Int. J. Pharm., 267, 79, (2003).

29. T. Vasconcelos, B. Sarmento, P. Costa, Drug Discovery Today, 12, 1068, (2007).

30. D. Q. M. Craig, Int. J. Pharm., 23, 131, (2002).

31. A. Newman, E. Munson, Amer. Pharm. Rev., 92, (2012). 